\title{
Communication-efficient and crash-quiescent Omega with unknown membership ${ }^{\text {th }}$
}

\author{
Sergio Arévalo ${ }^{\mathrm{a}}$, Ernesto Jiménez ${ }^{\mathrm{a}, *}$, Mikel Larrea $^{\mathrm{b}}$, Luis Mengual ${ }^{\mathrm{c}}$ \\ a EUI, Universidad Politécnica de Madrid, 28031 Madrid, Spain \\ b Universidad del País Vasco, 20018 San Sebastián, Spain \\ ' $\mathrm{FI}$, Universidad Politécnica de Madrid, 28660 Boadilla del Monte, Spain
}

\section{A R T I C L E I N F O}

Keywords:

Distributed computing

Fault tolerance

Unreliable failure detectors

\begin{abstract}
A B S T R A C T
The failure detector class Omega $(\Omega$ ) provides an eventual leader election functionality, i.e., eventually all correct processes permanently trust the same correct process. An algorithm is communication-efficient if the number of links that carry messages forever is bounded by $n$, being $n$ the number of processes in the system. It has been defined that an algorithm is crash-quiescent if it eventually stops sending messages to crashed processes. In this regard, it has been recently shown the impossibility of implementing $\Omega$ crash quiescently without a majority of correct processes. We say that the membership is unknown if each process $p_{i}$ only knows its own identity and the number of processes in the system (that is, $i$ and $n$ ), but $p_{i}$ does not know the identity of the rest of processes of the system. There is a type of link (denoted by ADD link) in which a bounded (but unknown) number of consecutive messages can be delayed or lost.

In this work we present the first implementation (to our knowledge) of $\Omega$ in partially synchronous systems with ADD links and with unknown membership. Furthermore, it is the first implementation of $\Omega$ that combines two very interesting properties: communication-efficiency and crash-quiescence when the majority of processes are correct. Finally, we also obtain with the same algorithm a failure detector $(\diamond \bar{P})$ such that every correct process eventually and permanently outputs the set of all correct processes.
\end{abstract}

\section{Introduction}

Unreliable failure detectors were proposed by Chandra and Toueg [4] as an elegant way of circumventing the well known FLP impossibility result [6] on solving deterministically the consensus problem [7] in crash-prone asynchronous environments. Informally, an unreliable failure detector provides hints about which processes are correct

\footnotetext{
* Research partially supported by the Spanish Research Council (MCeI) under grant TIN2010-19077, the Basque Government, under grants SPE09UN50 and IT395-10, and the Comunidad de Madrid, under grant S2009/TIC-1692.

* Corresponding author.

E-mail addresses: sergio.arevalo@eui.upm.es (S. Arévalo), ernes@eui.upm.es (E. Jiménez), mikel.larrea@ehu.es (M. Larrea), Imengual@fi.upm.es (L. Mengual).
}

and which ones are incorrect, i.e., have crashed so far. The failure detector can make mistakes, e.g., by erroneously suspecting a correct but slow process, or by not suspecting (yet) a crashed process. Nevertheless, to be useful it is required that failure detectors fulfill some completeness (i.e., suspect permanently crashed processes) and accuracy (i.e., stop suspecting correct processes) properties. For example, a failure detector such that every correct process eventually and permanently suspects all incorrect processes, and eventually does not suspect any correct process is named Eventually Perfect $(\diamond P)$.

Another important failure detector is Omega ( $\Omega$ ) [3], defined in terms of a trusted process, such that eventually all correct processes permanently trust the same correct process. It is said that $\Omega$ provides an eventual leader election functionality. This type of failure detector is important 
because it has been shown that $\Omega$ is the weakest failure detector for solving consensus [3].

We say that an algorithm implementing $\Omega$ is communication-efficient if the number of links that carry messages forever is bounded by $n$, being $n$ the number of processes in the system [2].

A new property called crash-quiescence has been recently proposed [9]. A distributed algorithm is said crashquiescent if it eventually stops sending messages to crashed processes. Sastry et al. have shown in [9] the impossibility of implementing $\Omega$ (and thus $\diamond P$ ) crash quiescently without a majority of correct processes in a partially synchronous system with links where a bounded, but unknown, number of consecutive messages can be arbitrarily late or lost (called ADD links). They also propose the first implementation of a (non-communication-efficient) $\diamond P$ algorithm that is crash-quiescent in any run with a majority of correct processes. ADD links [8] are interesting and realistic enough because they allow that an infinite number of messages could be lost or arbitrarily delayed, but guarantee that some subset of the messages sent on them will be received in a timely manner and such messages are not too sparsely distributed in time. In some sense, an ADD link successfully combines the losses property of a fair lossy link [1] (in which if an infinite number of messages are sent, then an infinite number of messages are received), and the timeliness property of an eventually timely link [1] (in which there is a time after which all the messages that are sent are received timely).

A system with unknown membership allows each process to execute the algorithm of $\Omega$ without having to know initially the identifiers of all processes (only knowing its own identifier and the total number of processes in the system is enough). Hence, you can execute the same code of $\Omega$ in runs with different processes without having to statically change the set of addresses of processes that participate in each run. This is possible because processes dynamically learn about the existence of other processes from the reception of messages. Note that this reduction of initial knowledge also supposes that if some process crashes before sending a message, the rest of processes will not be able to find out its existence as member of the system. For example, let us suppose you have a p2p environment in Internet where each server is identified by an IP-address. We know that the number of servers will be, for example, 10. In systems where the membership is known, each process has to know each of the 10 server's IP-address previously to start the execution of $\Omega$ to elect some of them as leader. In systems with unknown membership, the leader can be chosen without this knowledge by learning IP-addresses from the messages received from other processes.

Jiménez et al. show in [5] that it is necessary for each process to know the identity of the rest of processes of the system (that is, the membership) to implement a failure detector of anyone of the original eight classes proposed by Chandra and Toueg in [4] (and, hence, $\diamond P$ ). Interestingly, they also show in the same paper that $\Omega$ can be implemented without this knowledge. What it is possible to implement is the complement of $\diamond P$, that is, a failure detector such that every correct process eventually and permanently only trusts (instead of suspects) all correct (instead of incorrect) processes. We denote this failure detector as $\diamond \bar{P}$. Note that when the membership is known, $\diamond \bar{P}$ can be easily transformed into $\diamond P$ (we can obtain the set of suspected processes simply by removing from the set of all processes the set of trusted processes output by $\diamond \bar{P})$.

\subsection{Our results}

In this work we present the first implementation (to our knowledge) of $\Omega$ in partially synchronous systems with ADD links and where the membership is unknown. Furthermore, it is the first implementation that combines two very interesting properties: communication-efficiency and crash-quiescence when the majority of processes are correct. Finally, we also want to emphasize that the implementation presented in this paper also satisfies the properties of $\diamond \bar{P}$.

\section{The model}

We consider a system $S$ composed of a finite set $\Pi$ of $n$ processes. The process identifiers are totally ordered, but they do not need to be consecutive. We denote them by $p_{i}, p_{l}, p_{r}, \ldots$ We assume that processes only know about $\Pi$ their own identifier and what is the number $n$ of processes in $S$ (actually, they only need to known the majority, that is, $n / 2$ ). For example, process $p_{i}$ initially only knows $i$ and $n$.

Processes communicate only by sending and receiving messages. We assume that processes have a broadcasting primitive to send messages (called broadcast $(m)$ ). This primitive allows a process to send simultaneously the same message $m$ to the rest of processes in the system (that is, the same mechanism used in Ethernet or in IPmulticast). Furthermore, processes also have the possibility to use the primitive send (called send $(m)$ to $q$ ) to send the message $m$ to process $q$ only.

A process can fail by crashing permanently. We say that a process is correct if it does not fail, and we say a process is crashed (or it is a faulty process) if it fails. We use $C$ to denote the set of correct processes, and $F$ to denote the set of faulty processes. We consider that in a system $S$ there may be any number of crashed processes, and that this number is not known a priori. We assume that processes are synchronous in the sense that there are lower and upper bounds on the number of processing steps they execute per unit of time. These bounds are not known by processes. For simplicity, the local processing time is negligible with respect to message communication delays. Processes have timers that accurately measure intervals of time.

We assume that every pair of processes is connected by a pair of directed links. We consider that all links belong to type ADD (Average Delayed/Dropped) [8]. An ADD link allows an infinite number of messages to be lost or arbitrarily delayed, but guarantees that some subset of the messages sent on it will be received in a timely manner and such messages are not too sparsely distributed in time. More precisely, there are two unknown constants $\Delta$ and $B$ 


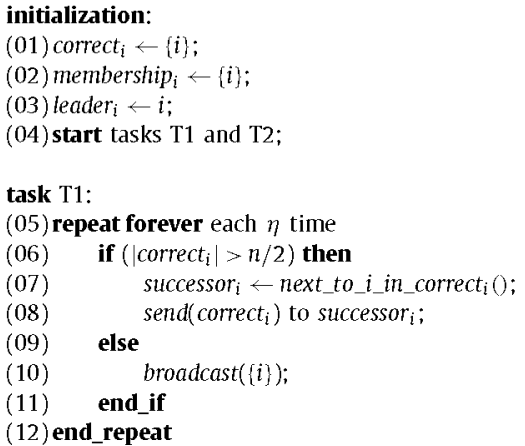

Fig. 1. Implementation of $\Omega$ (and $\diamond \bar{p}$ ) in a system where each process initially only knows its identity and $n$ (code for process $p_{i}$ ).

such that for all intervals of time in which a process $p_{i}$ sends at least $B$ messages to another process $p_{j}$, at least one of these messages is received by $p_{j}$ in at most $\Delta$ time.

\section{The Omega and $\diamond \bar{P}$ failure detectors}

Chandra et al. proposed the Omega $(\Omega)$ failure detector [3]. This failure detector guarantees that eventually all correct processes always have the same correct process as leader. More formally, the Omega failure detector satisfies the following property: there is a time after which each process $p_{i} \in C$ always has leader $r_{i}=p_{l}$, where $p_{l} \in C$.

Chandra and Toueg defined in [4] the Eventually Perfect $(\diamond P)$ failure detector. This failure detector guarantees that there is a time after which every correct process permanently only suspects all faulty processes. In [5] it is proven that if processes do not know a priori all members of the system (i.e., the membership is unknown), it is impossible to eventually suspect all faulty processes. For example, if we do not know the membership and process $p_{i}$ crashes at the very beginning of a run (before sending or receiving any message), it is not possible for another process $p_{j}$ to learn about $p_{i}$ 's presence in the system, and therefore, it is impossible for $p_{j}$ to suspect $p_{i}$.

Nevertheless, we can have in a system $S$ of our model a failure detector (we denote it as $\diamond \bar{P}$ ) such that there is a time after which every correct process permanently only trusts all correct processes. More formally, there is a time after which each process $p_{i} \in C$ always has $\mid$ correct $_{i}|=| C \mid$, and $p_{j} \in$ correct $_{i}$, for all $p_{j} \in C$. Note that in a system with known membership this failure detector can be easily transformed into $\diamond P$ (changing the output of each process $p_{i}$ by $\Pi \backslash$ correct $_{i}$ ). Interestingly, our protocol also implements $\diamond \bar{P}$.

\section{The implementation}

Fig. 1 implements $\Omega$ (and $\diamond \bar{P}$ ) in a system $S$ where the membership is unknown. If the majority of processes are correct, we will show that this protocol is communicationefficient and crash-quiescent.

In Fig. 1, each process $p_{i}$ initially broadcasts heartbeats repeatedly to indicate that it is alive (line 10 ). To know which processes are in the system, process $p_{i}$ has a set membership $_{i}$ (initially only contains itself). The set correct $_{i}$ maintains the processes it believes are alive (always containing at least itself). If a majority of processes are alive, then eventually $\mid$ correct $_{i} \mid>n / 2$ and process $p_{i}$ will send heartbeats periodically to successor ${ }_{i}$, instead of broadcast them (line 08). This variable successor ${ }_{i}$ contains the process returned by function next_to_i_in_correct ${ }_{i}($ ) (line 07). This function obtains the identifier of the process closest to the identifier of $p_{i}$ in the sequence formed by all elements of correct $_{i}$, in increasing order and cyclic (that is, like operation mod but with the elements of correct $_{i}$ instead of a subset of natural numbers). For example, if process $p_{3}$ has correct $_{3}=\{1,3,5,8\}$, then next_to_i_in_correct 3() will return 5 . In another example, if process $p_{3}$ has correct co $_{3}=$ $\{1,2,3\}$, then next_to_i_in_correct 3() will return 1 . As we will show, if $\mid$ correct $_{i} \mid>n / 2$, each correct process $p_{i}$ will eventually send messages only to one correct process, establishing a cycle (we will define it below as ring) formed by all correct processes. The variable leader $_{i}$ has the identifier of the process that $p_{i}$ considers its leader. Its value is the smallest value in correct $_{i}$.

\section{Correctness proof}

Let $D$ be a subset of correct processes $(D \subseteq C)$. We say that there is a relation $p_{i} \rightarrow p_{j}, p_{i}, p_{j} \in D$, if there is a time after which process $p_{i}$ is permanently sending heartbeats to $p_{j}$. We say that $p_{i}$ is the predecessor of $p_{j}$, and $p_{j}$ is the successor of $p_{i}$ in $D$. We say there is a ring among all processes in $D$, denoted by $\operatorname{ring}(D)$, if each process $p_{i} \in D$ has a unique predecessor and a unique successor with respect to all processes in $D$. For example, if $D$ contains the subset of correct processes $\left\{p_{l}, p_{k}, p_{r}, p_{s}\right\}$, and $p_{r} \rightarrow p_{k} \rightarrow p_{l} \rightarrow p_{s} \rightarrow p_{r}$, then we say that there is a $\operatorname{ring}(D)$.

The following lemma states that eventually crashed processes cannot be in the set correct $i$ of any correct process $p_{i}$.

Lemma 1. For every process $p_{i} \in C$, there is a time after which if $j \in$ correct $_{i}$, then $p_{j} \in C$. 
Proof. Let us consider a process $p_{j} \in F$. We prove that eventually and permanently $j \notin$ correct $_{i}, \forall p_{i} \in C$. Let $s e q_{j}$ be a permutation of the processes in $C$, where the first element of the sequence $s e q_{j}$ is the process whose identifier next_to_i_in_correct ${ }_{j}()$ would return if correct $_{j}=C$. Let $\operatorname{seq}_{j}(x)$ be, for $x \geqslant 1$, the $x$ th element of the sequence $s e q_{j}$. The element $s e q_{j}(x), x \geqslant 2$, is the process whose identifier next_to_i_in_correct seq $_{j}(x-1) 0$ would return if correct $_{\text {seq }_{j}(x-1)}=$ C.

We are going to prove that, for all $x \geqslant 1$, eventually and permanently $j \notin$ correct $_{\text {seq }_{j}(x)}$, by induction on $x$. Base case is $x=1$. Let us consider a time $\tau$ at which all faulty processes are crashed, and all messages sent by these processes have disappeared from the system (i.e., they have been delivered or lost). After $\tau$, process $\operatorname{seq}_{j}$ (1) will never receive any heartbeat with a set containing $j$. This is so, because a process sending a heartbeat by execution of line 10, it only includes its own identifier (which cannot be $j$ since process $p_{j}$ is already crashed). On the other hand, if the heartbeat is sent by execution of line 08 , the values in this set correct $_{i}, \forall p_{i} \in C$, sent to process seq $_{j}(1)$ do not include $j$ (otherwise, the heartbeat would have been sent to $p_{j}$ instead). For example, let us consider that $j=2, \Pi=\left\{p_{0}, p_{1}, p_{2}, p_{3}\right\}, p_{j} \in F$ and $t>\tau$. We know that $\operatorname{seq}_{2}=3 \mapsto 0 \mapsto 1 \mapsto 2$ (where $a \mapsto b$ denotes that $a$ precedes to $b$ in the sequence), and, hence, $\operatorname{seq}_{2}(1)=3$. Let us also consider that at time $t>\tau$ we have correct $_{0}=\{2,0\}$ and correct $t_{1}=\{3,0,1\}$. Then, process $p_{0}$ sends its heartbeats to process $p_{2}$ (which is crashed), and process $p_{1}$ sends its heartbeats to process $p_{3}$ (and as base case states, correct $_{1}$ does not contain 2). If $j \in$ correct $_{\text {seq }_{j}(1)}$ at time $\tau$, eventually the timer $\operatorname{timer}_{\text {seq }_{j}(1)}(j)$ will expire and $j$ will be permanently removed from correct $_{\text {Seq }_{j}(1)}$. If $j \notin$ correct $_{\text {seq }}(1)$ at time $\tau, j$ is never included after $\tau$. Hence, there is a time $\tau_{1} \geqslant \tau$ after which $j \notin$ correct $_{\text {seq }_{j}(1)}$ permanently.

Let us now consider $x \geqslant 2$. By induction hypothesis, after $\tau_{x-1}$, we have that $j \notin$ correct $_{y}$, for all $y \in\{1, \ldots, x-1\}$. Note that now process $s e q_{j}(x)$ will receive heartbeats from seq $_{j}(x-1)$ with a set correct $_{\text {seq }_{j}(x-1)}$ not containing $j$. Then, following the same reasoning of the base case, process $\operatorname{seq}_{j}(x)$ will never receive any heartbeat with a set containing $j$. Hence, there is a time $\tau_{x} \geqslant \tau_{x-1}$ after which $j \notin$ correct $_{\text {Seq }_{j}(x)}$ permanently.

Therefore, for all $x \geqslant 1$, eventually and permanently $j \notin$ correct $_{s e q_{j}(x)}$. As the sequence $\operatorname{seq}_{j}$ includes all correct processes, and the proof holds for all $p_{j} \in F$, the lemma follows.

The following lemma assures that if eventually a correct process $p_{i}$ believes that a minority of processes are alive, then eventually all correct processes in the system will believe that $p_{i}$ is alive.

Lemma 2. If there is a process $p_{i} \in C$ such that eventually $\mid$ correct $_{i} \mid \leqslant n / 2$, then there is a time after which $i \in$ correct $_{j}$, for every $p_{j} \in C$.

Proof. If there is a time after which a process $p_{i} \in C$ always has $\mid$ correct $_{i} \mid \leqslant n / 2$, then it will be permanently broadcasting heartbeats with $i$ each $\eta$ time (line 10). As all links are ADD, then at least one from each $B$ messages is receive in each process $p_{j}$ in at most $\eta B+\Delta$ time. Hence, eventually timer ${ }_{j}(i)$ will never expire, and process $p_{j}$ will always have $i \in$ correct $_{j}$.

The following lemma shows that if a majority of processes are crashed, eventually all correct processes have $\mid$ correct $\mid \leqslant n / 2$.

Lemma 3. If $|C| \leqslant n / 2$, then there is a time after which $\mid$ correct $_{i} \mid \leqslant n / 2$, for every process $p_{i} \in C$.

Proof. From Lemma 1, there is a time after which every correct process $p_{i} \in C$ will have $\mid$ correct $_{i}|\leqslant| C \mid$. Hence, if $|C| \leqslant n / 2$, then $\mid$ correct $_{i} \mid \leqslant n / 2$.

In the following lemma we show that if there is a minority of correct processes, eventually all correct processes have the same set correct.

Lemma 4. If $|C| \leqslant n / 2$, there is a time after which correct $t_{i}=$ correct $_{j}$, for all $p_{i}, p_{j} \in C$.

Proof. From Lemma 3 , if $|C| \leqslant n / 2$, there is a time after which every correct process $p_{i}$ will broadcast heartbeats permanently with its identifier $i$ each $\eta$ time (line 10). Then, eventually no heartbeats with $j$, where $p_{j} \in F$, will be received by correct processes. Then, $\operatorname{timer}_{i}(j)$ eventually will expire (if it was previously set by $p_{i}$ ) and $j$ will not be in correct $_{i}$ anymore. Hence, there is a time after which correct $_{i}$ does not contain identifiers of crashed processes. From Lemma 2 and Lemma 3, if $|C| \leqslant n / 2$, there is a time after which every $p_{i} \in C$ will have $j \in$ correct $_{i}$, for every process $p_{j} \in C$. Therefore, if $|C| \leqslant n / 2$, there is a time after which correct $_{i}=$ correct $_{j}$, for all $p_{i}, p_{j} \in C$.

The following lemma shows that if a minority of processes are crashed, eventually all correct processes have $\mid$ correct $\mid>n / 2$.

Lemma 5. If $|C|>n / 2$, then there is a time after which $\mid$ correct $_{i} \mid>n / 2$, for every process $p_{i} \in C$.

Proof. By contradiction, let us suppose that $|C|>n / 2$, and there is a subset $E \neq \emptyset$ such that eventually each process $p_{k} \in E$ has $\mid$ correct $_{k} \mid \leqslant n / 2$. Then, there is also a complementary subset $G$ such that eventually each process $p_{l} \in G$ has $\mid$ correct $_{l} \mid>n / 2$ (being $E \cap G=\emptyset$, and $E \cup G=C$ ). We have two cases to study:

Case 1. $G=\emptyset$. That is, every correct process $p_{k} \in E$. From Lemma 2, there is a time after which $k \in$ correct $_{j}$, for every $p_{j} \in C$. So, as by hypothesis of contradiction $|C|>n / 2$, we eventually have $\mid$ correct $_{j} \mid>n / 2$, for every $p_{j} \in C$, and hence, we reach a contradiction.

Case 2. $G \neq \emptyset$. By hypothesis of contradiction we also have that $E \neq \emptyset$. Note that eventually each process $p_{l} \in G$, as it has $\mid$ correct $_{l} \mid>n / 2$, sends heartbeats permanently with 
correct $t_{l}$ each $\eta$ time to its successor (line 08). As links are $A D D$, then at least one from each $B$ messages will be received by every correct process in at most $\eta B+\Delta$ time. Hence, there is a time after which the successor of $p_{l}$ will receive on time heartbeats of $p_{l}$. From Lemma 1 , eventually correct $_{i}$ can only contain correct processes, for every process $p_{i} \in C$. From Lemma 2, there is a time after which each process $p_{j} \in C$ has $k \in$ correct $_{j}$, for every process $p_{k} \in$ $E$. Note that for each process its set correct is updated with the values received from heartbeats (line 20). Hence, eventually the successor of some process $p_{g} \in G$ will be one of the processes $p_{s}$ of $E$. So, eventually correct $g \subseteq$ correct $_{s}$, and as $\mid$ correct $_{g} \mid>n / 2$, we also have that $\mid$ correct $_{s} \mid>n / 2$, and we reach a contradiction.

The following lemma proves that if the majority of processes are correct, a unique ring is formed among all of them.

Lemma 6. If $|C|>n / 2$, then there is a unique $\operatorname{ring}(C)$.

Proof. If there is a time after which some process $p_{i} \in C$ has $\mid$ correct $_{i} \mid>n / 2$, then eventually process $p_{i}$ sends heartbeats with correct $_{i}$ each $\eta$ time to its successor (line 08). As links are ADD, then at least one from each $B$ messages will be received by every correct process in at most $\eta B+\Delta$ time. So, there is a time after which the successor of $p_{i} \in C$ will receive on time heartbeats of $p_{i}$.

From Lemma 1 , eventually correct $t_{i}$ can only contain correct processes, for every process $p_{i} \in C$. From Lemma 5 , if $|C|>n / 2$ then eventually $\mid$ correct $_{i} \mid>n / 2$, for every process $p_{i} \in C$. So, process $p_{i}$ will form a ring with other correct processes, and the intersection between each pair of rings is not empty. More formally, let us suppose that there are $\mathrm{m}$ subsets of correct processes, denoted by $C_{1}, C_{2}, \ldots, C_{m}$, such that: (a) $\operatorname{ring}\left(C_{1}\right), \operatorname{ring}\left(C_{2}\right), \ldots, \operatorname{ring}\left(C_{m}\right)$, (b) $C_{1} \cup C_{2} \cup$ $\cdots \cup C_{m}=C$, and $(\mathrm{c})$ ring $\left(C_{1}\right) \cap$ ring $\left(C_{2}\right) \cap \cdots \cap$ ring $\left(C_{m}\right) \neq \varnothing$.

Let us use induction in the number $n$ of rings from each process $p_{a} \in \operatorname{ring}\left(C_{k}\right)$ and each process $p_{b} \in \operatorname{ring}\left(C_{k+n}\right)$ to show that, if $|C|>n / 2$, then there is a unique $\operatorname{ring}(C)$.

Let $n=1$, that is, $p_{a}, p_{b} \in \operatorname{ring}\left(C_{k}\right)$. The claim is trivially true.

Let us now suppose that the claims holds for any distance $0 \leqslant n \leqslant r$. Let us now prove that the claim is also held for $n+1$. We have process $p_{a} \in \operatorname{ring}\left(C_{k+n}\right)$, process $p_{b} \in \operatorname{ring}\left(C_{k+n+1}\right)$, and, from the induction hypothesis we have that $|C|>n / 2$, and hence, from Lemmas 1 and 5 , the intersection between these two rings is not empty. That is, there is some correct process $p_{x}$ such that $p_{x} \in \operatorname{ring}\left(C_{k+n}\right)$, and $p_{x} \in \operatorname{ring}\left(C_{k+n+1}\right)$. Hence, process $p_{x}$ receives the set correct of some process $p_{s} \in \operatorname{ring}\left(C_{k+n}\right)$ and some process $p_{t} \in \operatorname{ring}\left(C_{k+n+1}\right)$ (i.e., process $p_{x}$ is the successor of $p_{s}$ and $p_{t}$ ). As correct process $p_{x}$ updates its set correct $_{x}$ with the values received from heartbeats (line 20), it eventually sends its set correct $t_{x}$ with the union of correct from $\operatorname{ring}\left(C_{k+n}\right)$ and $\operatorname{ring}\left(C_{k+n+1}\right)$. Hence, a unique ring will be formed among all correct processes of both rings. Therefore, if $|C|>n / 2$, then there is a unique $\operatorname{ring}(C)$.
In the following lemma we show that if there is a majority of correct processes, eventually all correct processes have the same set correct.

Lemma 7. If $|C|>n / 2$, there is a time after which correct $_{i}=$ correct ${ }_{j}$, for all $p_{i}, p_{j} \in C$.

Proof. From Lemma 6 , if $|C|>n / 2$, there is ring $(C)$. From Lemma 1, the set correct $t_{i}$ of each process $p_{i} \in C$ eventually can only contain correct processes. Then, each process $p_{i} \in$ $C$ sends correct $t_{i}$ to its successor (line 08 ). This successor of $p_{i}$ (for example $p_{k}$ ) includes the processes sent by process $p_{i}$ in its set correct $_{k}$ (line 20). Hence, as there is a ring, eventually correct $_{i}=$ correct $_{j}$, for all $p_{i}, p_{j} \in C$. Therefore, if $|C|>n / 2$, there is a time after which correct $_{i}=$ correct $_{j}$, for all $p_{i}, p_{j} \in C$.

Theorem 1. Let process $p_{l} \in C$. There is a time after which every process $p_{i} \in C$ permanently has leader $r_{i}=l$.

Proof. From Lemma 1, there is a time after which all processes in correct $_{i}$ have to be correct, for every $p_{i} \in C$. From Lemmas 4 and 7 , there is a time after which correct $_{i}=$ correct $_{j}$, for every $p_{i}, p_{j} \in C$. Note that correct $t_{i}$ is never empty, for every process $p_{i}$. This is so because initially process $p_{i}$ includes itself in this set (line 01), and this value $i$ is never removed from correct ${\text { (because } \text { timer }_{i}(i)}$ is never started, lines 14 and 21). Hence, there is a time after which process $p_{i}$ permanently has leader $_{i}=l$, being $l=\min \left(\right.$ correct $\left._{i}\right)$ (lines 23 and 27).

Theorem 2. If $|C|>n / 2$, the algorithm of Fig. 1 is communication-efficient and crash-quiescent.

Proof. From Lemmas 5 and 6 , there is a $\operatorname{ring}(C)$ if $|C|>$ $n / 2$. Then, this ring will be formed by sending heartbeats permanently among all correct processes in a cyclic way, and thus, the number of links that eventually carry messages forever is $|C|$. Hence, the algorithm of Fig. 1 is communication-efficient and crash-quiescent.

In the following theorem we show that the algorithm of Fig. 1 also implements $\diamond \bar{P}$.

Theorem 3. There is a time after which each process $p_{i} \in C$ always has $\mid$ correct $_{i}|=| C \mid$, and $p_{j} \in$ correct $_{i}$, for all $p_{j} \in C$.

Proof. From Lemma 1, eventually crashed processes cannot be in the set correct $i, \forall p_{i} \in C$.

If $|C| \leqslant n / 2$, from Lemmas 2 and 3 , eventually each process $p_{i} \in C$ has $j \in$ correct $_{i}, \forall p_{j} \in C$. Hence, if $|C| \leqslant n / 2$, there is a time after which each process $p_{i} \in C$ always has $\mid$ correct $_{i}|=| C \mid$, and $p_{j} \in$ correct $_{i}$, for all $p_{j} \in C$.

If $|C|>n / 2$, from Lemma 7 we have that eventually correct $_{i}=$ correct $_{j}$, for all $p_{i}, p_{j} \in C$, and from Lemma 6 there is a unique ring formed by all correct processes. Looking at the algorithm of Fig. 1, each process $p_{i}$ of this unique ring sends heartbeats to its successor (i.e., process $p_{j}$ ) with correct $_{i}$ (line 08), and this successor $p_{j}$ includes the values of correct $_{i}$ in its correct ${ }_{j}$ (lines 14 and 20). We 
know that correct $_{i}$ always contains at least $i$, for all process $p_{i}$. This is so because initially process $p_{i}$ includes itself in this set (line 01), and this value $i$ is never re-

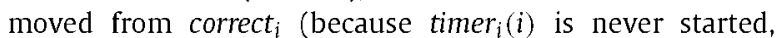
lines 14 and 21). Hence, if $|C|>n / 2$, there is a time after which each process $p_{i} \in C$ always has $\mid$ correct $_{i}|=| C \mid$, and $p_{j} \in$ correct $_{i}$, for all $p_{j} \in C$.

Therefore, there is a time after which each process $p_{i} \in C$ always has $\mid$ correct $_{i}|=| C \mid$, and $p_{j} \in$ correct $_{i}$, for all $p_{j} \in C$.

\section{References}

[1] M. Aguilera, C. Delporte-Gallet, H. Fauconnier, S. Toueg. On imple menting omega with weak reliability and synchrony assumptions, in: Proceedings of the 22nd ACM Symposium on Principles of Distributed Computing, PODC 2003, Boston, Massachusetts, USA, July 2003, pp. 306-314.

[2] M. Aguilera, C. Delporte-Gallet, H. Fauconnier, S. Toueg, Stable leader election, in: Proceedings of the 15th International Symposium on Dis tributed Computing (DISC'2001), Lisbon, Portugal, October 2001, in: LNCS, vol. 2180, Springer-Verlag, 2001, pp. 108-122.

[3] T.D. Chandra, V. Hadzilacos, S. Toueg, The weakest failure detector for solving consensus, Journal of the ACM 43 (4) (July 1996) 685-722.

[4] T.D. Chandra, S. Toueg, Unreliable failure detectors for reliable distributed systems, Journal of the ACM 43 (2) (March 1996) 225-267.

[5] E. Jiménez, S. Arévalo, A. Fernández, Implementing unreliable failure detectors with unknown membership, Information Processing Letters 100 (2) (October 2006) 60-63.

[6] M. Fischer, N. Lynch, M. Paterson, Impossibility of distributed consensus with one faulty process, Journal of the ACM 32 (2) (April 1985) 374-382.

[7] M. Pease, R. Shostak, L. Lamport, Reaching agreement in the presence of faults, Journal of the ACM 27 (2) (April 1980) 228-234.

[8] S. Sastry, S. Pike, Eventually perfect failure detection using ADD chan nels, in: Proceedings of the 5th International Symposium on Parallel and Distributed Processing and Applications, ISPA 2007, Niagara Falls, Canada, August 2007, in: LNCS, vol. 4742, Springer-Verlag, 2007. pp. 483-496.

[9] S. Sastry, S. Pike, J. Welch, Crash-quiescent failure detection, in: Proceedings of the 23rd International Symposium on Distributed Computing, DISC2009, Elche, Spain, September 2009, in: LNCS, vol. 5805, Springer-Verlag, 2009, pp. 326-340. 